\title{
Search for Supersymmetric Particles Using Acoplanar Charged-Particle Pairs from $\mathrm{Z}^{0}$ Decays
}

\author{
1 December 1989
}

\section{The ALEPH Collaboration}

D. Decamp, B. Deschizeaux, J.-P. Lees, M.-N. Minard Laboratoire de Physique des Particules (LAPP), IN ${ }^{2} P^{3}$-CNRS, 74019 Annecy-le-Vieux Cedex, France

J.M. Crespo, M. Delfino, E. Fernandez ${ }^{1}$, M. Martinez, R. Miquel, Ll.M. Mir, S. Orteu, A. Pacheco, J.A. Perlas, E. Tubau Laboratorio de Fisica de Altas Energias, Universidad Autonoma de Barcelona, 08193 Bellaterra (Barcelona), Spain ${ }^{10}$

M.G. Catanesi, M. de Palma, A. Farilla, G. Iaselli, G. Maggi, A. Mastrogiacomo, S. Natali, S. Nuzzo, A. Ranieri, G. Raso, F. Romano, F. Ruggieri, G. Selvaggi, L. Silvestris, P. Tempesta, G. Zito INFN Sezione di Bari e Dipartimento di Fisica dell' Università, 70126 Bari, Italy

Y. Chen, H. Hu, D. Huang, J. Lin, T. Ruan, T. Wang, W. Wu, Y. Xie, D. Xu, R. Xu, J. Zhang, W. Zhao Institute of High-Energy Physics, Academia Sinica, Beijing, The People's Republic of China ${ }^{11}$

H. Albrecht ${ }^{2}$, F. Bird, E. Blucher, T. Charity, H. Drevermann, Ll. Garrido, C. Grab, R. Hagelberg, S. Haywood, B. Jost, M. Kasemann, G. Kellner, J. Knobloch, A. Lacourt, I. Lehraus, T. Lohse, D. Lüke ${ }^{2}$, A. Marchioro, P. Mato, J. May, V. Mertens, A. Minten, A. Miotto, P. Palazzi, M. Pepe-Altarelli, F. Ranjard, J. Richstein ${ }^{3}$, A. Roth, J. Rothberg ${ }^{4}$ H. Rotscheidt, W. von Rüden, R. St.Denis, D. Schlatter, M. Takashima, M. Talby, H. Taureg, W. Tejessy, H. Wachsmuth, S. Wheeler, W. Wiedenmann, W. Witzeling, J. Wotschack European Laboratory for Particle Physics (CERN), 1211 Geneva 23, Switzerland

Z. Ajaltouni, M. Bardadin-Otwinowska, A. Falvard, P. Gay, P. Henrard, J. Jousset, B. Michel, J-C. Montret,

D. Pallin, P. Perret, J. Prat, J. Proriol, F. Prulhière Laboratoire de Physique Corpusculaire, Université Blaise Pascal, IN ${ }^{2} P^{3}$-CNRS, Clermont-Ferrand, 63177 Aubière, France

H. Bertelsen, F. Hansen, J.D. Hansen, J.R. Hansen, P.H. Hansen, A. Lindahl, B. Madsen, R. Mø llerud, B.S. Nilsson, G. Petersen Niels Bohr Institute, 2100 Copenhagen, Danmark ${ }^{12}$

E. Simopoulou, A. Vayaki Nuclear Research Center Demokritos (NRCD), Athens, Greece

J. Badier, D. Bernard, A. Blondel, G. Bonneaud, J. Bourotte, F. Braems, J.C. Brient, M.A. Ciocci, G. Fouque,

R. Guirlet, P. Miné, A. Rougé, M. Rumpf, H. Videau, I. Videau ${ }^{1}$, D. Zwierski Laboratoire de Physique Nucléaire et des Hautes Energies, Ecole Polytechnique, IN ${ }^{2} P^{3}-C N R S, 91128$ Palaiseau Cedex, France

(Submitted to Physics Letters B)

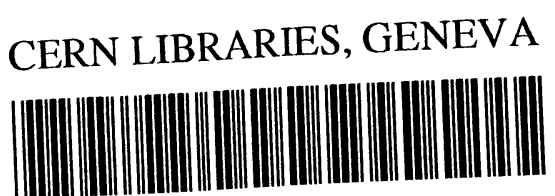


D.J. Candlin

Department of Physics, University of Edinhurgh, Edinburgh EH9 3JZ, United Kingdom ${ }^{13}$

A. Conti, G. Parrini

Dipartimento di Fisica, Università di Firenze, INFN Sezione di Firenze, 50125 Firenze, Italy

M. Corden, C. Georgiopoulos, J.H. Goldman, M. Ikeda, J. Lannutti, D. Levinthal ${ }^{18}$, M. Mermikides, L. Sawyer Supercomputer Computations Research Institute and Dept. of Physics, Florida State University, Tallahassee FL 32306, USA ${ }^{151617}$

A. Antonelli, R. Baldini, G. Bencivenni, G. Bologna ${ }^{5}$, F. Bossi, P. Campana, G. Capon, V. Chiarella, G. De Ninno, B. D'Ettorre-Piazzoli ${ }^{6}$, G. Felici, P. Laurelli, G. Mannocchi ${ }^{6}$, F. Murtas, G.P. Murtas, G. Nicoletti, P. Picchi ${ }^{5}$, P. Zografou Laboratori Nazionali dell'INFN (LNF-INFN), 00044 Frascati, Italy

B. Altoon, O. Boyle, A.J. Flavell, A.W. Halley, I. ten Have, J.L. Hearns, I.S. Hughes, J.G. Lynch, D.J. Martin, R. O'Neill, C. Raine, J.M. Scarr, K. Smith ${ }^{1}$, A.S. Thompson Department of Physics and Astronomy, University of Glasgow, Glasgow G12 8QQ, United Kingdom ${ }^{13}$

B. Brandl, O. Braun, R. Geiges, C. Geweniger ${ }^{1}$, P. Hanke, V. Hepp, E.E. Kluge, Y. Maumary, M. Panter, A. Putzer, E. Rensch, A. Stahl, K. Tittel, M. Wunsch Institut für Hochenergiephysik, Universität Heidelberg, 6900 Heidelberg, Fed. Rep. of Germany ${ }^{19}$

A.T. Belk, R. Beuselinck, D.M. Binnie, W. Cameron ${ }^{1}$, M. Cattaneo, P.J. Dornan, A.M. Greene, S. Dugeay, R.W. Forty, J.F. Hassard, S. Patton, J.K. Sedgbeer, G. Taylor, I.R. Tomalin, A.G. Wright Department of Physics, Imperial College, London SWT 2BZ, United Kingdom ${ }^{13}$

P. Girtler, D. Kuhn, G. Rudolph Institut für Experimentalphysik, Universität Innsbruck, 6020 Innsbruck, Austria ${ }^{21}$

C.K. Bowdery ${ }^{1}$, T.J. Brodbeck, A.J. Finch, F. Foster, G. Hughes, N.R. Keemer, M. Nuttall, B.S. Rowlingson, T. Sloan, S.W. Snow Department of Physics, University of Lancaster, Lancaster LA1 4YB, United Kingdom ${ }^{13}$

T. Barczewski, L.A.T. Bauerdick, K. Kleinknecht ${ }^{1}$, B. Renk, S. Roehn, H.-G. Sander, M. Schmelling, F. Steeg Institut für Physik, Universität Mainz, 6500 Mainz, Fed. Rep. of Germany ${ }^{10}$

J-P. Albanese, J-J. Aubert, C. Benchouk, A. Bonissent, F. Etienne, R. Nacasch, P. Payre, B. Pietrzyk ${ }^{1}$, Z. Qian Centre de Physique des Particules, Faculté des Sciences de Luminy, IN ${ }^{2} P^{3}-C N R S, 13288$ Marseille, France

W. Blum, P. Cattaneo, M. Comin, G. Cowan, B. Dehning, H. Dietl, M. Fernandez-Bosman, D. Hauff, A. Jahn, E. Lange, G. Lütjens, G. Lutz, W. Männer, H-G. Moser, Y. Pan, R. Richter, A.S. Schwarz, R. Settles, U. Stiegler, U. Stierlin, G. Stimpfl ${ }^{7}$, J. Thomas, G. Waltermann Max-Planck-Institut für Physik und Astrophysik, Werner-Heisenberg-Institut für Physik, 8000 München, Fed. Rep. of Germany ${ }^{10}$

G. de Bouard, J. Boucrot, O. Callot, X. Chen, A. Cordier, M. Davier, G. Ganis, J.-F. Grivaz, Ph. Heusse, P. Janot, V. Journé, D.W. Kim, J. Lefrançois, D. Lloyd-Owen, A.-M. Lutz, P. Marotte, J.-J. Veillet Lalboratoire de l'Accéléateur Linéaire, Université de Paris-Sud, IN ${ }^{2} P^{3}$-CNRS, 91405 Orsay Cedex, France 
S.R. Amendolia, G. Bagliesi, G. Batignani, L. Bosisio, U. Bottigli, C. Bradaschia, I. Ferrante, F. Fidecaro, L. Foà ${ }^{1}$, E. Focardi, F. Forti, A. Giassi, M.A. Giorgi, F. Ligabue, A. Lusiani, E.B. Mannelli, P.S. Marrocchesi, A. Messineo, F. Palla, G. Sanguinetti, S. Scapellato, J. Steinberger, R. Tenchini, G. Tonelli, G. Triggiani Dipartimento di Fisica dell'Università, INFN Sezione di Pisa, e Scuola Normale Superiore,56010 Pisa, Italy

J.M. Carter, B.J. Green, M.G. Green, A.K. McKemey, P.V. March, T. Medcalf, M.R. Saich, J.A. Strong ${ }^{1}$, R.M. Thomas, T. Wildish Department of Physics, Royal Holloway \& Bedford New College, University of London, Surrey TW20 OEX, United Kingdom ${ }^{13}$

D.R. Botterill, R.W. Clifft, T.R. Edgecock, M. Edwards, S.M. Fisher, J. Harvey, D.L. Hill, T.J. Jones, M. Morrissey, P.R. Norton, D.P. Salmon, J.C. Thompson High-Energy Physics Division, Rutherford Appleton Laboratory, Chilton, Didcot, OXON OX11 OQX, United Kingdom ${ }^{13}$

B. Bloch-Devaux, P. Colas, C. Klopfenstein, E. Lançon, E. Locci, S. Loucatos, L. Mirabito, E. Monnier, P. Perez, F. Perrier, J. Rander, J.-F. Renardy, A. Roussarie, J.-P. Schuller Département de Physique des Particules Élémentaires, CEN-Saclay, 91191 Gif-sur-Yvette Cedex, France $^{20}$

J.G. Ashman, C.N. Booth, F. Combley, M. Dinsdale, J. Martin, D. Parker, L.F. Thompson Department of Physics, University of Sheffield, Sheffield S3 7RH, United Kingdom ${ }^{13}$

S. Brandt, H. Burkhardt, C. Grupen, H. Meinhard, E. Neugebauer, U. Schäfer, H. Seywerd, K. Stupperich Fachbereich Physik, Universität Siegen, 5900 Siegen, Fed. Rep. of Germany ${ }^{10}$

B. Gobbo, F. Liello, E. Milotti, F. Ragusa ${ }^{8}$, L. Rolandi ${ }^{1}$

Dipartimento di Fisica, Università di Trieste e INFN Sezione di Trieste, 34127 Trieste, Italy

L. Bellantoni, J.F. Boudreau, D. Cinabro, J.S. Conway, D.F. Cowen, Z. Feng, J.L. Harton, J. Hilgart, R.C. Jared ${ }^{9}$, R.P. Johnson, B.W. LeClaire, Y.B. Pan, T. Parker, J.R. Pater, Y. Saadi, V. Sharma, J.A. Wear, F.V. Weber, Sau Lan Wu, S.T. Xue, G. Zobernig

Department of Physics, University of Wisconsin, Madison, WI 53706, USA ${ }^{14}$

\footnotetext{
${ }^{1}$ Now at CERN.

${ }^{2}$ Permanent address: DESY, Hamburg, Fed. Rep. of Germany.

${ }^{3}$ Now at LeCroy, Geneva.

${ }^{4}$ On leave of absence from University of Washington, Seattle, WA 98195.

${ }^{5}$ Also Istituto di Fisica Generale, Università di Torino, Torino, Italy.

${ }^{6}$ Also Istituto di Cosmo-Geofisica del C.N.R., Torino, Italy.

${ }^{7}$ Now at FSU.

${ }^{8}$ Now at INFN Milano.

${ }^{9}$ Permanent address: LBL, California, USA.

${ }^{10}$ Supported by CAICYT, Spain.

${ }^{11}$ Supported by the National Science Foundation of China.

${ }^{12}$ Supported by the Danish Natural Science Research Council.

${ }^{13}$ Supported by the UK Science and Engineering Research Council.

${ }^{14}$ Supported by the US Department of Energy, contract DE-AC102-76ER00881.

${ }^{15}$ Supported by the US Department of Energy, contract DE-FG05-87ER40319.

${ }^{16}$ Supported by the NSF, contract PHY-8451274.

${ }^{17}$ Supported by the US Department of Energy, contract DE-FCOS-85ER250000.

${ }^{18}$ Supported by SLOAN fellowship, contract BR 2703.

${ }^{19}$ Supported by the Bundesministerium für Forschung und Technologie, Fed. Rep. of Germany.

${ }^{20}$ Supported by the Institut de Recherche Fondamentale du C.E.A..

${ }^{21}$ Supported by Fonds zur Förderung der wissenschaftlichen Forschung, Austria.
} 


\begin{abstract}
We have performed a search for supersymmetric particles using acoplanar pairs of oppositely-charged particles in decays of the $\mathrm{Z}^{0}$. In $0.53 \mathrm{pb}^{-1}$ of integrated luminosity near the $\mathrm{Z}^{0}$ peak, we observe two events where approximately four are expected from background, allowing limits to be extended on combined photino and slepton masses, and also on combined photino and chargino masses.
\end{abstract}




\section{Introduction}

Several extensions to the Standard Model [1] of electroweak interactions have been proposed to help explain its shortcomings, two of which are the "naturalness" problem (in which the mass of the Higgs must be fine-tuned to be kept at the electroweak scale) and the related "hierarchy" problem (where the mass of the Higgs boson has large corrections due to any new mass scale) [2]. One way to overcome these problems is to introduce a symmetry between bosons and fermions, known as supersymmetry [3]. If such a symmetry exists then every fermion (boson) would have a bosonic (fermionic) partner. If supersymmetry were unbroken the masses of a particle and its superpartner would be equal. The empirical absence of such equal-mass partners implies that either supersymmetry is badly broken or is not a symmetry of nature.

We describe here a search for $\mathrm{Z}^{0}$ decays to pairs of oppositely-charged particles which are acoplanar (that is, the plane of the charged particles does not include the beam axis) and which have missing energy. Such decays are a signature of the production of pairs of the supersymmetric partners of the known leptons and pairs of charginos, the superpartners of the $\mathrm{W}^{ \pm}$and Higgs bosons [4]. These particles are expected to decay immediately to their associated particles and a photino. The photino is assumed here to be the lightest supersymmetric particle, to be stable (as expected from R-parity conservation [5]), and to interact weakly with matter [6]. Thus it escapes detection, carrying away transverse momentum and energy.

\section{Production of Sleptons and Charginos}

Figure 1 shows the production processes we consider here and the decay modes of the supersymmetric particles. The production of a pair of supersymmetric scalar leptons (sleptons, denoted as $\tilde{e}, \tilde{\mu}, \tilde{\tau}$ ) can occur through the s-channel exchange of a $\mathrm{Z}^{0}$ or photon, and also through the t-channel exchange of a photino in the case of scalar electron production as shown in Figure $1 \mathrm{~b}$. At the $\mathrm{Z}^{0}$ peak the contribution from the t-channel is less than $10 \%$, and we include it in the result presented here. The cross section depends on the masses of the left- and right-handed sleptons, which may or may not be equal. We present below the limits on these masses for the cases $m_{\tilde{I}_{L}}=m_{\tilde{I}_{R}}$ 
and $m_{\tilde{\mathfrak{l}}_{L}} \gg m_{\tilde{I}_{R}}$, which differ by roughly a factor of two in cross section. As depicted in Figure 1c, the sleptons decay to their associated leptons and a photino.

Pair production of charginos $\tilde{\chi}^{ \pm}$, which are the mass eigenstates of admixtures of wino-like and higgsino-like "current" eigenstates, also proceeds through the s-channel as shown in Figure 1a. Though no model-independent prediction for the admixture exists, the couplings of the current eigenstates are predicted exactly. We present below our result for the limiting cases of a pure wino and a pure higgsino; the light chargino in any model will lie between these extremes. The coupling of the wino to the $\mathrm{Z}^{0}$ is nearly an order of magnitude larger than that of the higgsino [7]. For a pure wino, the cross section at the $\mathrm{Z}^{0}$ pole is on the order of $10 \mathrm{nb}$, and decreases slowly with increasing wino mass. In this search we are only sensitive to decay modes resulting in one charged particle per chargino. Such final states arise from from three-body decays to a lepton, its associated neutrino, and a photino (or, more generally, the lightest neutralino [4]) as shown in Figure 1d. We do not consider here the case where the scalar neutrino is lighter than the chargino. For simplicity only decays to electrons and muons in the final state are used to set mass limits; the branching ratios of a chargino to each of these is taken to be $1 / 9$, the minimum of the range given in the model [8].

All the cross section calculations below are made with the assumptions of the minimal supersymmetric standard model [4], [9]. The Born-order cross section is modified strongly by initial-state radiation; at the $\mathrm{Z}^{0}$ pole the cross section decreases by approximately $30 \%$ if the effect is included. We employ here the method of Berends and Kleiss [10] to determine the cross section including this effect.

\section{The Apparatus}

The ALEPH detector is described elsewhere [11], but we briefly describe here the components relevant to this measurement. The central charged particle tracking detectors are an axial-wire drift chamber (ITC) at small radii and a large cylindrical time projection chamber (TPC). The ITC provides trigger information and up to 8 coordinates in azimuth and radius while the TPC provides up to 21 three-dimensional coordinates along the trajectory of a charged particle. The momentum of charged particles is de- 
termined from the curvature of the particle trajectory in the 1.5 Tesla magnetic field from the superconducting solenoid. Between the TPC and the coil cryostat lies the electromagnetic calorimeter (ECAL), which is a lead sheet/proportional wire chamber sandwich having 74000 cathode pad towers in a projective geometry. The 45 wire planes in each of the 36 modules are also read out individually and are used in triggering. At small angles is a similarly-constructed electromagnetic calorimeter, the LCAL, used to measure the luminosity. Finally, the iron return yoke of the solenoid houses the limited streamer tube chambers of the hadronic calorimeter (HCAL), used to measure the energy of hadrons and also to trigger on and identify muons.

Data were collected at seven energies around the $\mathrm{Z}^{0}$ peak, from $88.3 \mathrm{GeV}$ to 94.3 GeV in steps of $1 \mathrm{GeV}$, during the first main running period of the LEP machine. Half of the total integrated luminosity of $0.53 \mathrm{pb}^{-1}$ was collected at the energy nearest the peak, $91.3 \mathrm{GeV}$. A total of 11133 hadronic events (five or more charged tracks carrying at least $10 \%$ of the center-of-mass energy) were observed in the sample.

\section{Event Selection and Background}

Our event selection has two steps, and serves a twofold purpose. Firstly, we wish to select a sample of clean two-track events satisfying basic criteria discussed below. These events can then be compared with our simulation of the well-known Standard Model processes from which they come. Secondly, we wish to apply further criteria to eliminate as many as possible of the coplanar events from these known processes, while maintaining as good efficiency for the acoplanar signal events as possible. The number of events passing our criteria is then used to determine limits on the production of supersymmetric particles.

Our most basic criterion is that there be two and only two tracks from oppositelycharged particles in the tracking detectors. Tracks are required to originate from a point less than $7 \mathrm{~cm}$ from the interaction point along the beam axis and less than $3 \mathrm{~cm}$ in radius from the beam axis, have an angle of greater than $18.2^{\circ}$ with the beam axis, have at least 5 TPC coordinates, and have momentum greater than $100 \mathrm{MeV}$.

To eliminate events which are acoplanar due to accompanying high-energy photons, 
events are rejected if there are any ECAL energy deposits over $5 \mathrm{GeV}$ which are more than $25.4^{\circ}$ away from the tracks' initial directions. Also, to eliminate events with large initial-state radiation at small angles, or two-photon events with high-energy smallangle beam particles, we require that there be no energy deposit of $5 \mathrm{GeV}$ or more in any of the four modules of the luminosity calorimeter.

The 2151 remaining two-track events are almost all due to the processes

$$
\begin{aligned}
& e^{+} e^{-} \rightarrow e^{+} e^{-}(\gamma) \\
& e^{+} e^{-} \rightarrow \mu^{+} \mu^{-}(\gamma) \\
& e^{+} e^{-} \rightarrow \tau^{+} \tau^{-}(\gamma) \\
& e^{+} e^{-} \rightarrow e^{+} e^{-} e^{+} e^{-} \\
& e^{+} e^{-} \rightarrow e^{+} e^{-} \mu^{+} \mu^{-} \\
& e^{+} e^{-} \rightarrow e^{+} e^{-} \tau^{+} \tau^{-} \\
& e^{+} e^{-} \rightarrow e^{+} e^{-} \text {(hadrons) }
\end{aligned}
$$

The first three processes are production of lepton pairs from $\mathrm{Z}^{0}$ decay, and the last four are two-photon processes in which the final-state "beam" $e^{ \pm}$escape through the holes in the apparatus for the beam pipe. We have simulated production of $1.5 \mathrm{pb}^{-1}$ of each of the above processes [12], including all particle interaction effects (scattering, bremsstrahlung, energy loss, etc.) and the specific geometry of the sensitive and insensitive regions of our detector. The efficiency of the trigger is determined using the data, and incorporated in the simulation. The simulation uses the same event reconstruction program as the real data.

We can compare the events passing our criteria with the simulated events. To determine the normalization we use the number of hadronic events in the runs from which the sample is selected. Figure 2a compares the distribution of the acoplanarity angle for events with both tracks in the barrel region of the detector. The acoplanarity angle is defined as the difference in azimuthal angle of the two tracks. Clearly the events are very coplanar, which is to be expected from dilepton events and from twophoton events with no activity detected in the LCAL from the scattered beam particles. The agreement between data and simulation is very good both in normalization and in shape. Figure $2 b$ shows a comparison of the distribution of the missing energy. The 
high end is dominated by the two-photon processes, the middle region is dominated by tau-pair decays, and the peak near zero is from $\mu$ - and e-pair events. The shape in the peak disagrees with the simulation due to as-yet-uncorrected drift field distortions in the TPC. These distortions, however, do not significantly affect the analysis.

In the second step of our event selection we wish to eliminate events from the background processes, while keeping the signal events. The signal we seek is characterized by two high-energy charged particles which are acoplanar, and missing energy. To eliminate the Standard Model background we require

- momentum of each track $>2 \mathrm{GeV}$

- $18.2^{\circ}<$ acoplanarity angle $<161.8^{\circ}$

- $10 \mathrm{GeV}<$ missing energy $<80 \mathrm{GeV}$

The momentum and acoplanarity requirements reduce the number of background events to thirteen, and after the missing energy requirement we are left with two events. The simulation predicts $4.1 \pm 0.3$ background events; our observation is consistent with this. Thus, following the procedure for setting limits on a Poisson process with background outlined in Ref. [13], we can exclude at the $95 \%$ confidence level any signal process for which we would have seen more than 4.2 signal events. This value is used in determination of mass limits below.

\section{Mass Limits on Supersymmetric Particles}

We now use the result of the previous section to obtain limits on the masses of supersymmetric particles. To estimate our efficiency for signal detection we use the simulation as described in the previous section. The good agreement of the simulation with our data gives us confidence in applying it to an unobserved process. We use all beam energies and corresponding integrated luminosities determined from the number of hadronic events to calculate the number of events which would pass the same criteria as applied to the data.

Figure 3a shows the region of the $m_{\tilde{e}}-m_{\tilde{\gamma}}$ plane excluded by our search to $95 \%$ confidence for the two cases $m_{\tilde{e}_{L}}=m_{\tilde{e}_{R}}$ and $m_{\tilde{e}_{L}} \gg m_{\tilde{e}_{R}}$. In the degenerate-mass 
case we exclude scalar electrons with mass less than $43.5 \mathrm{GeV}$ and roughly $5 \mathrm{GeV}$ greater than the photino mass for photino masses up to $35 \mathrm{GeV}$. Previous limits for the degenerate-mass case from the TOPAZ [14] and ASP [15] experiments are shown. The ASP result is a $90 \%$ confidence limit.

The production of a pair of smuons proceeds through exactly the same mechanism as selectron-pair production, except that there is no contribution from t-channel processes. Figure $3 \mathrm{~b}$ shows the excluded mass region, which covers nearly the entire kinematically accessible region; for the degenerate-mass case smuons with mass less than $42.6 \mathrm{GeV}$ and approximately $5 \mathrm{GeV}$ greater than that of the photino are excluded for a photino mass of up to $33 \mathrm{GeV}$. The result from TOPAZ [14] for the degenerate-mass case is also shown.

Mass limits have also been obtained by L3 [16] for selectrons and smuons, to 40 and $41 \mathrm{GeV}$ respectively at a photino mass of $20 \mathrm{GeV}$.

For the production of pairs of scalar taus, which also proceeds only through the s-channel, we take advantage of the $85 \%$ branching ratio into single charged particles. The efficiency in this channel is lower, however, due to the softer momentum spectrum and the presence of additional photons from $\pi^{0}$ 's. Hence we obtain a smaller excluded mass region, shown in Figure 3c along with the region excluded by TOPAZ [14]. In the degenerate-mass case we exclude the region of scalar tau mass below $40.4 \mathrm{GeV}$ and about $5 \mathrm{GeV}$ above the photino mass for photino masses up to $15 \mathrm{GeV}$.

For chargino pairs we arrive at the limits shown in Figure 4, which cover the mass region up to $45.5 \mathrm{GeV}$ in the pure wino case, and to $44.5 \mathrm{GeV}$ in the limit of a pure higgsino, for photino masses of up to 35 and $30 \mathrm{GeV}$ respectively. The result from the TOPAZ [14] experiment at $m_{\tilde{\gamma}}=0 \mathrm{GeV}$ excluded a pure wino below $26.9 \mathrm{GeV}$, and L3 [16] has excluded a wino with mass less than $42 \mathrm{GeV}$. (Other experiments [15] [17] exclude winos in a similar mass range, under the assumption of a scalar neutrino lighter than the wino.) 


\section{Conclusions}

We have searched over $10^{4}$ decays of the $\mathrm{Z}^{0}$ for two acoplanar charged particles with missing energy, and find two candidates satisfying our criteria where we expected approximately four from Standard Model background processes. From this observation we have excluded to $95 \%$ confidence regions of the scalar lepton-photino mass plane and the chargino-photino mass plane, where the scalar lepton or chargino is several $\mathrm{GeV}$ heavier than the photino such that it decays to final states including a photino. For the sleptons $\tilde{e}, \tilde{\mu}$, and $\tilde{\tau}$, the high-mass edges of the excluded regions are $43.5 \mathrm{GeV}$, $42.6 \mathrm{GeV}$, and $40.4 \mathrm{GeV}$, respectively, and are roughly independent of photino mass. A pure wino of mass less than $45.5 \mathrm{GeV}$ is excluded, and a pure higgsino with mass less than $44.5 \mathrm{GeV}$ is excluded, both roughly independent of photino mass up to $28 \mathrm{GeV}$. These results significantly extend the excluded regions in these parameters.

\section{Acknowledgements}

We would like to thank our colleagues of the LEP division for their outstanding performance in bringing the LEP machine into operation. Thanks are also due to the many engineering and technical personnel at CERN and at the home institutes for their contribution toward ALEPH's success. Those of us not from member states wish to thank CERN for its hospitality.

\section{References}

[1] S.L. Glashow, Nucl. Phys. 22 (1961) 579; S. Weinberg, Phys. Rev. Lett. 19 (1967) 1264; A. Salam, Elementary Particle Theory, ed. N. Svartholm, Stockholm, (Almquist and Wiksell, 1968), 367.

[2] C.H. Llewellyn Smith and G.G. Ross, Phys. Lett. 105B (1981) 38.

[3] J. Wess and B. Zumino, Phys. Lett. 49B (1974) 52; for a review see P. Fayet and S. Ferrara, Phys. Rep. 32C (1977) 249. 
[4] H.E. Haber and G.L. Kane, Phys. Rep. 117, Nos. 2-4 (1985) 75-263 and references therein.

[5] P. Fayet, "Unification of the Fundamental Particle Interactions", Proc. Europhys. Study Conf., Erice (Plenum, 1980) p. 587.

[6] P. Fayet, Phys. Lett. 86B (1979) 272.

[7] P. Fayet, Phys. Lett. 133B (1983) 363.

[8] T. Schimert, C. Burgess, and X. Tata, Phys. Rev. D32 (1985) 707; T. Schimert and X. Tata, Phys. Rev. D32 (1985) 721.

[9] H. Nilles, Phys. Rep. C10 (1984) 1, and references therein; R. Barbieri, Riv. Nuovo. Cim. 11 (1988); M. Chen, C. Dionisi, M. Martinez, and X. Tata, Phys. Rep. 159 (1988) 201.

[10] G. Altarelli, R. Kleiss, and C. Verzegnassi, eds., Z Physics at LEP 1, CERN Report 89-08 (1989), vol. III, p. 18 ff. and references therein.

[11] “ALEPH - a Detector for electron-positron Annihilation at LEP," paper in preparation for publication in Nucl. Instr. Meth.

[12] For electron-pair production we used the BABAMC Monte Carlo; M. Bohm, A. Denner, and W. Hollik, Nucl. Phys. B304 (1988) 687, and F.A. Berends, R. Kleiss, and W. Hollik, Nucl. Phys. B304 (1988) 712. For $\mu$-and $\tau$-pair production we used the KORALZ program; S. Jadach, et al., Z Physics at LEP 1, CERN Report 89-08 (1989), vol. III, p. 69 ff. For two-photon processes leading to dilepton final states the program DIAG36 was used; see F.A. Berends et al. Nucl. Phys. B253 (1985) 441. For two-photon processes leading to hadronic final states we used the PHOT01 Monte Carlo, based on the work of J.A.M. Vermasseren, with later contributions from S. Kawabata, J. Olsson, H. Wriedt, and J.M. Nye.

[13] Particle Data Group, Phys. Lett. 204B (1988) 81; O. Helene, Nucl. Instr. and Meth. 212 (1983) 319.

[14] I. Adachi et al., Phys. Lett. 218B (1989) 105. 
[15] C. Hearty et al., Phys. Rev. D39 (1989) 3207.

[16] "Mass limits for scalar smuons, scalar electrons, and winos from $\mathrm{e}^{+} \mathrm{e}^{-}$collisions near $\sqrt{s}=91 \mathrm{GeV}$ ", L3 Collaboration, submitted to Phys. Lett. B. (L3 Preprint 2)

[17] R. Ansari, et al., Phys. Lett. 195B (1987) 613. 


\section{List of Figures}

Figure 1. Processes studied in this search: $a$, pair production of sleptons and charginos from $\mathrm{Z}^{0}$ exchange; $b$, t-channel production of scalar electrons; $c$, decay of scalar leptons to associated lepton and photino; $d$, three-body decays of charginos.

Figure 2. Comparison of data and simulated distributions: $a$, acoplanarity angle distribution; $b$, missing energy distribution. Here we require that the momentum be greater than $1 \mathrm{GeV}$, the invariant mass of the two-track system be greater than $2 \mathrm{GeV}$, and each track have a polar angle in the range $53^{\circ}$ to $127^{\circ}$.

Figure 3. Region in combined slepton and photino masses excluded to $95 \%$ confidence by this search: $a$, result for scalar electron pair production with previous results from TOPAZ [14] and ASP [15] (the result from ASP is to 90\% confidence); $b$, result for scalar muon pair production with previous result from TOPAZ; $c$, result for scalar tau pair production with previous result from TOPAZ.

Figure 4. Region in combined chargino and photino masses excluded to $95 \%$ confidence by this search for the extremes of a pure wino and a pure higgsino. 
(a)

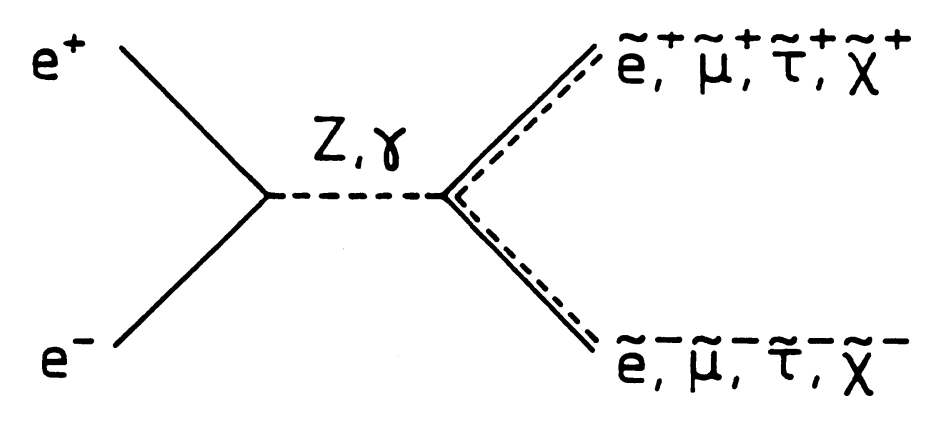

(b)

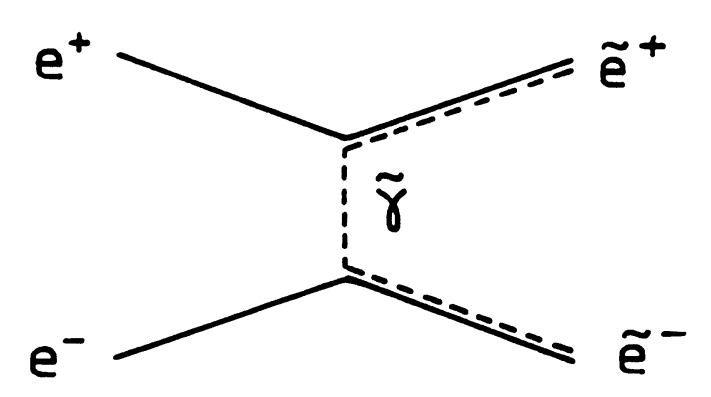

(c)

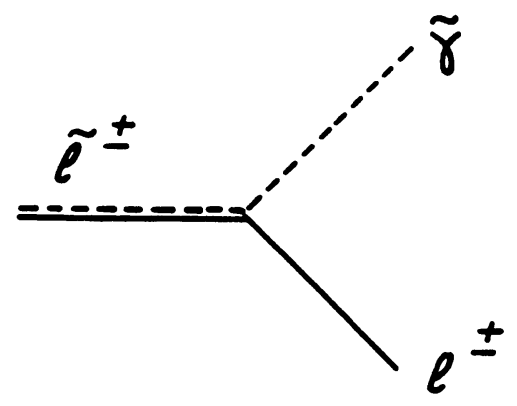

(d)
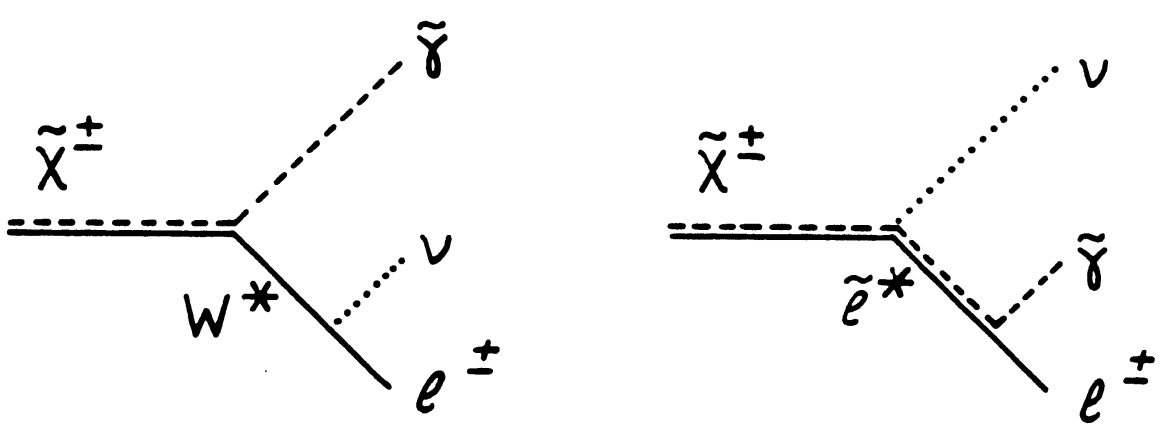

Fig. 1 


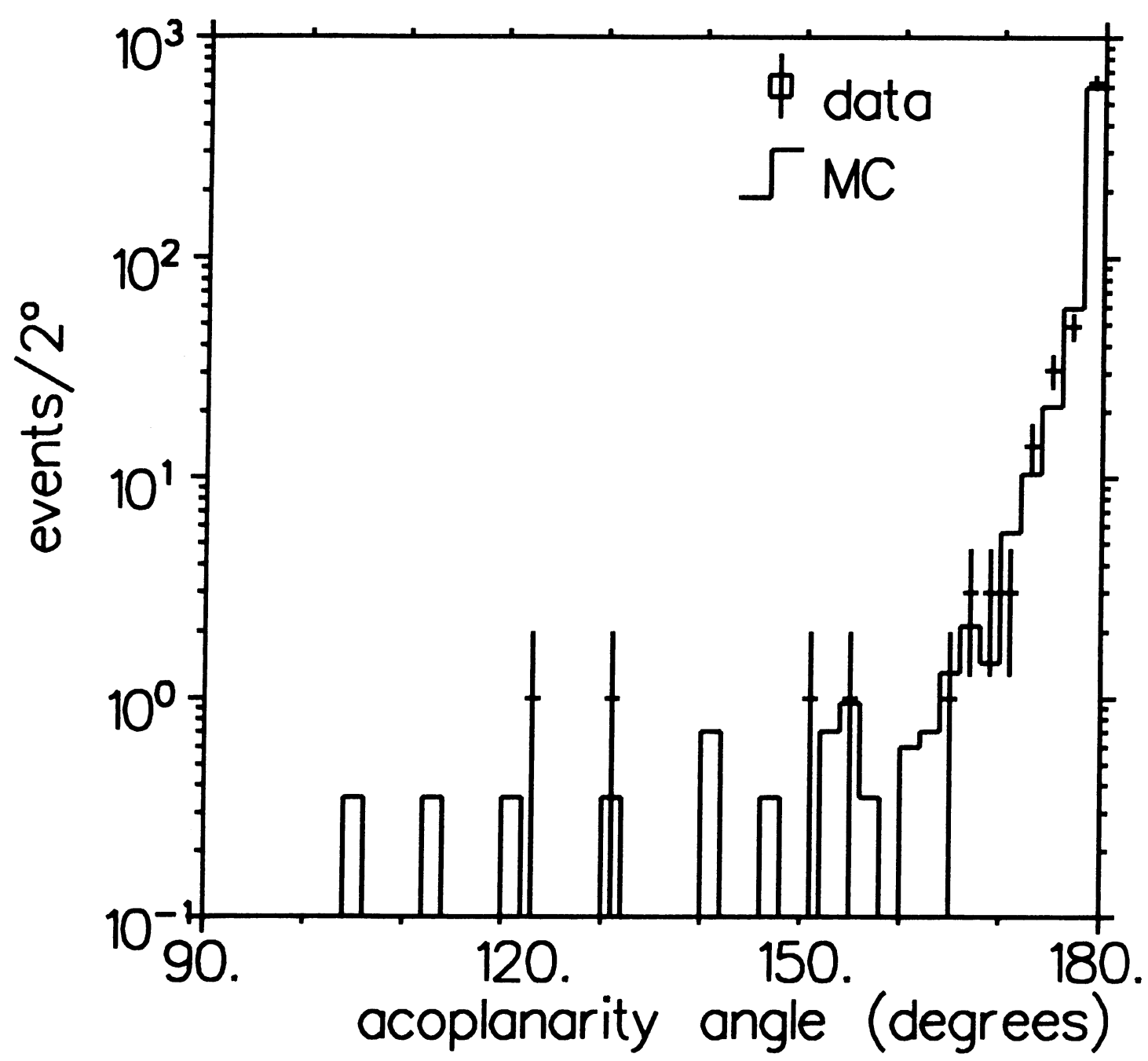

Fig. 2a 


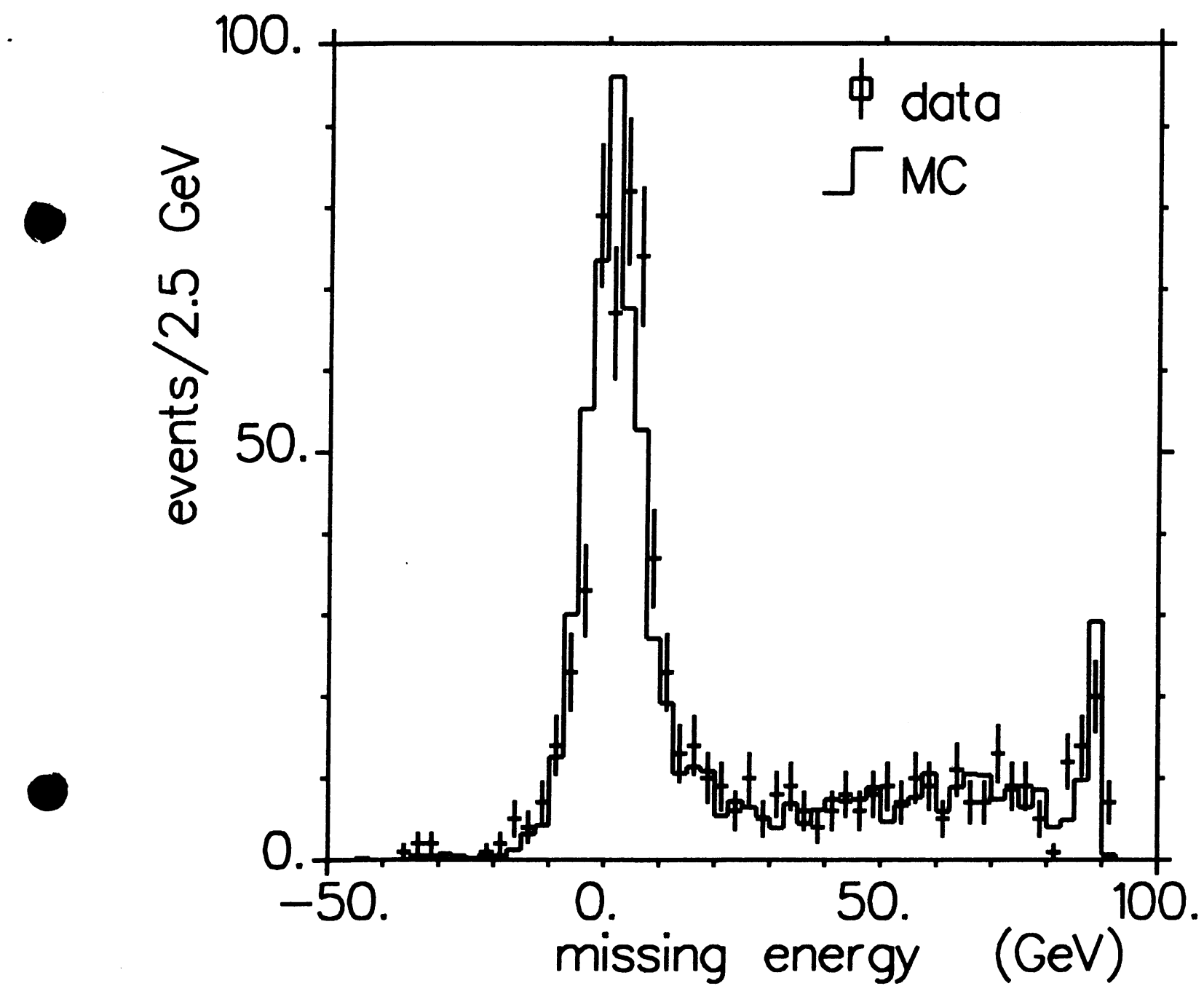

Fig. 2b 


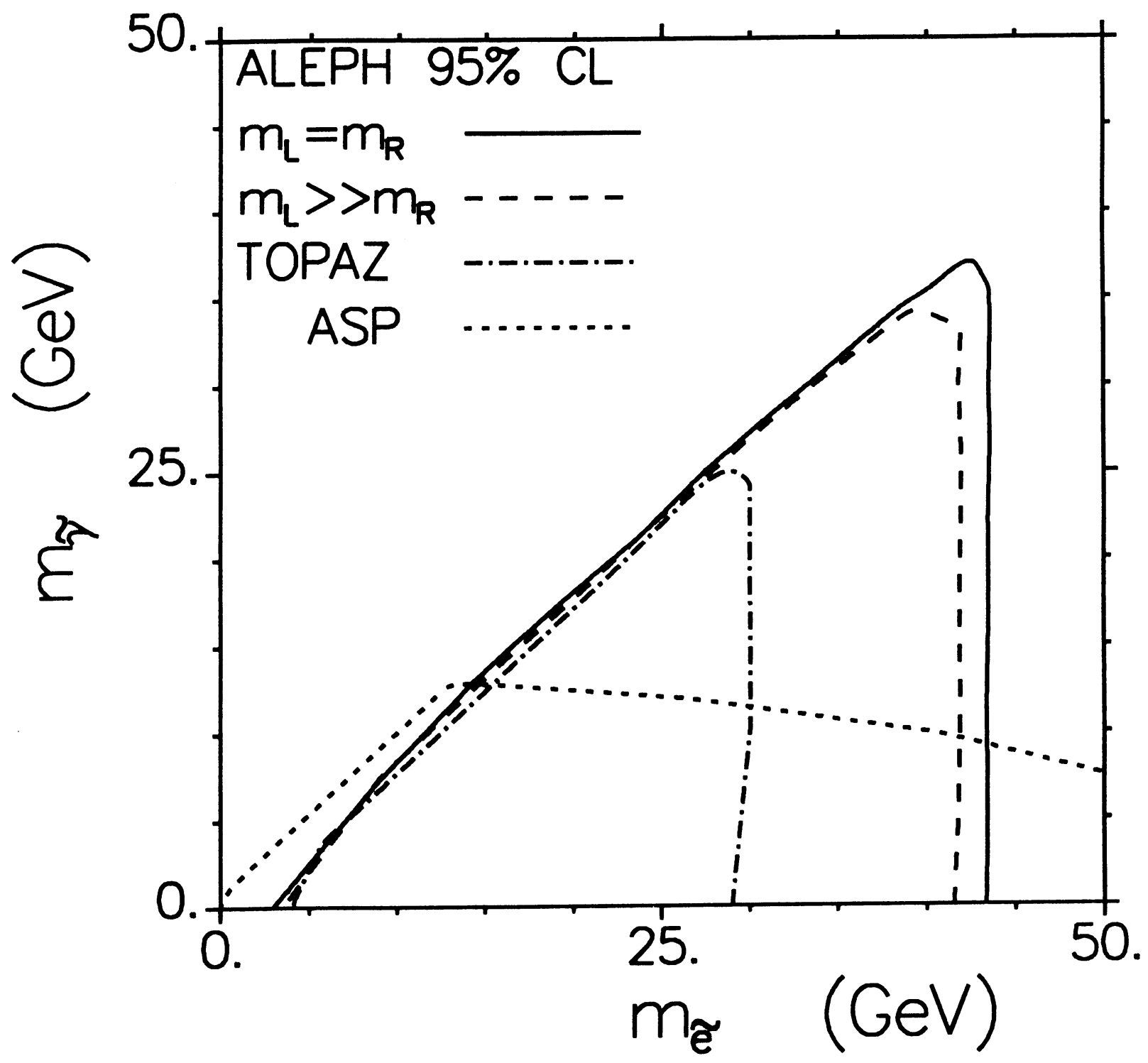

Fig. 3a 


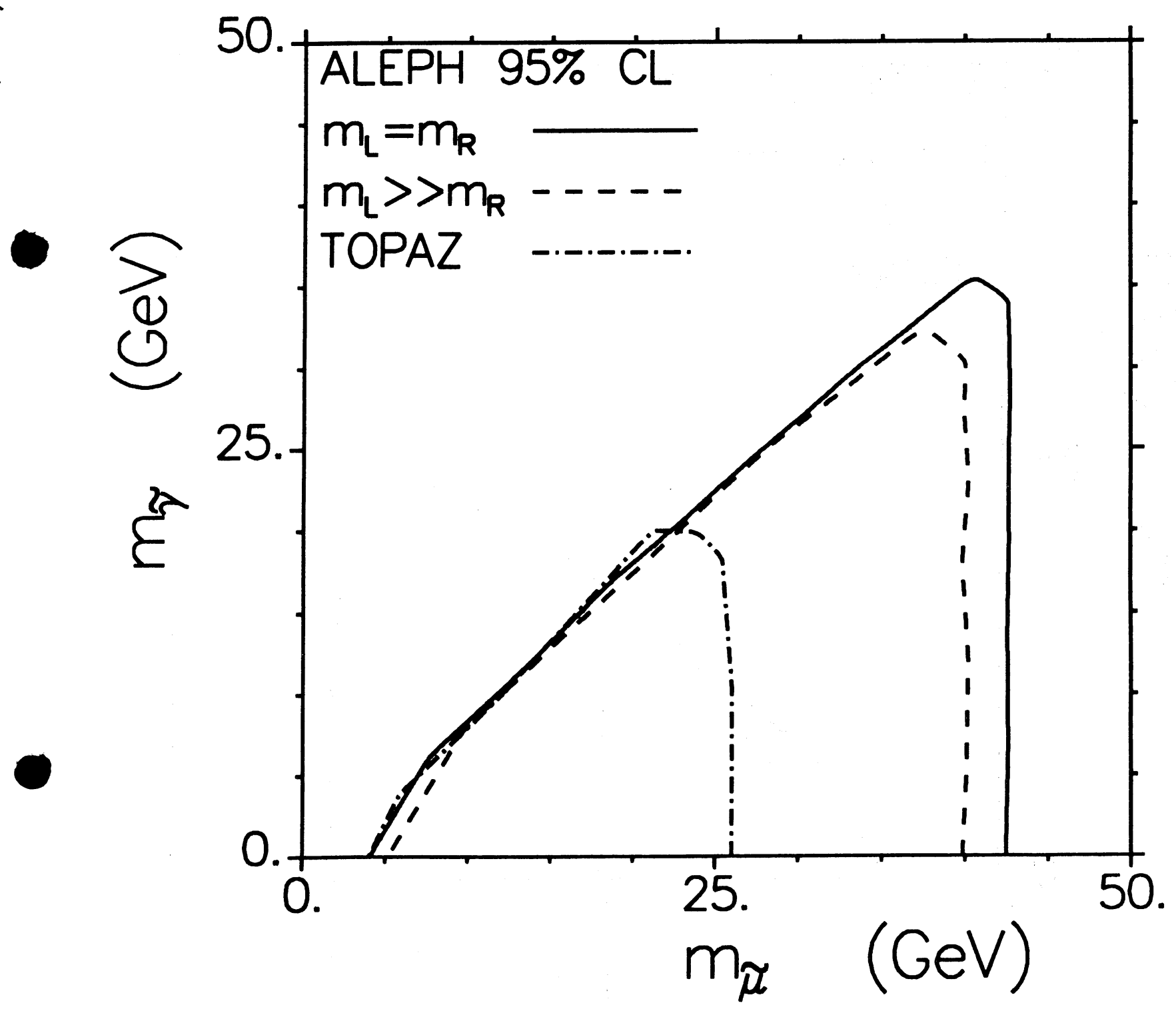

Fig. 3b 


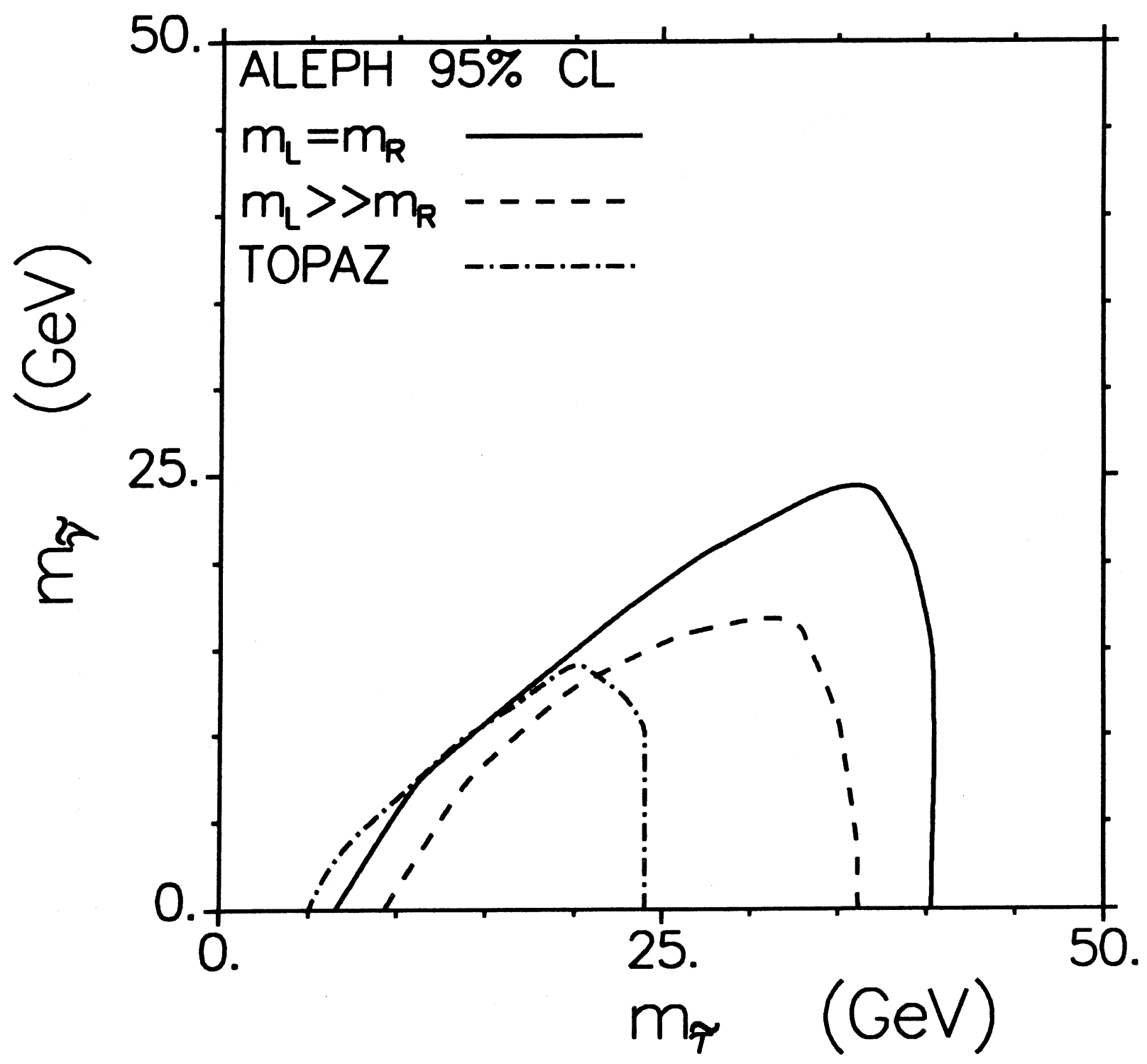

Fig. 3c 


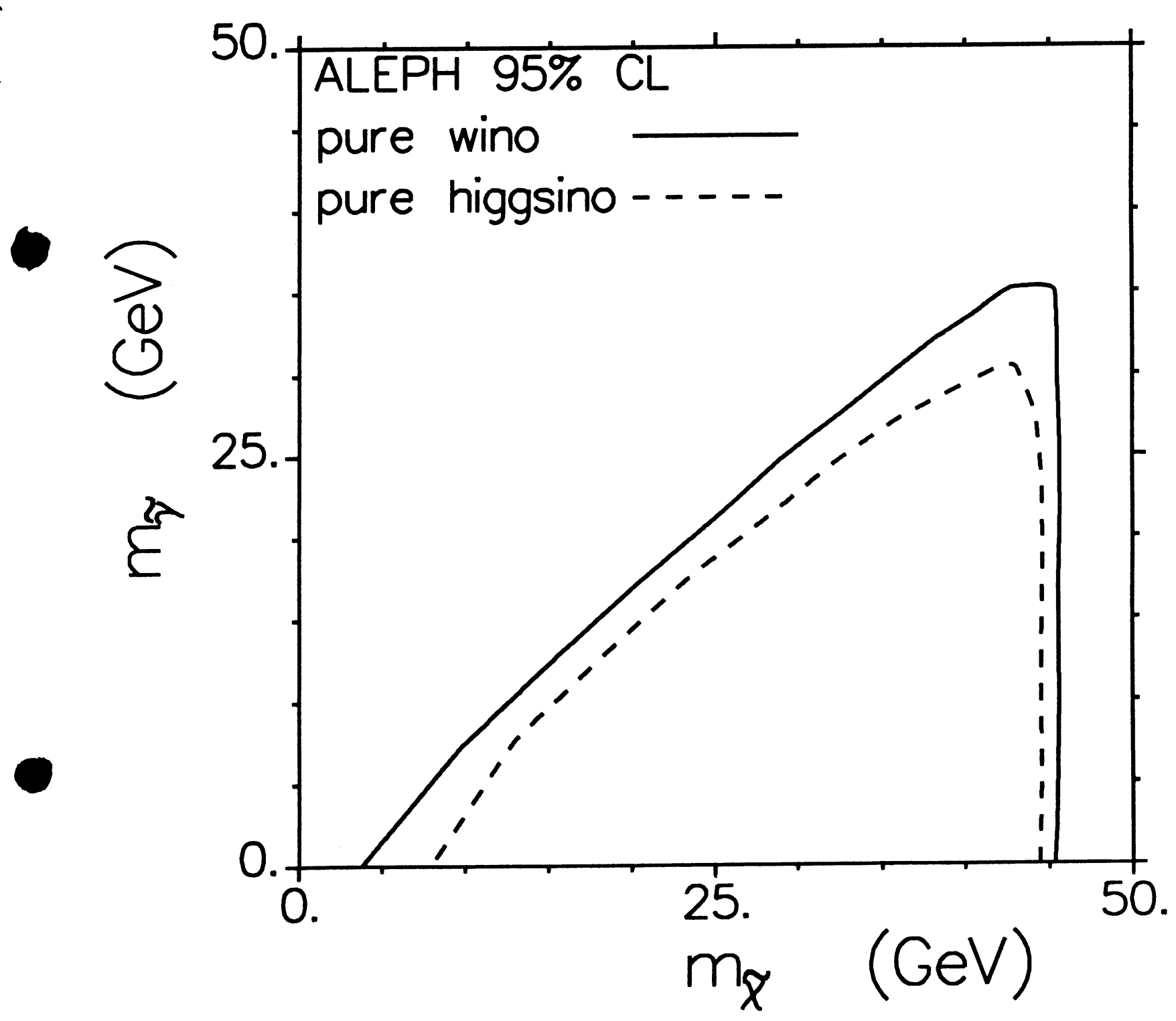

Fig. 4 